\title{
Risk for Thyroid Cancer Recurrence Is Higher in Men Than in Women Independent of Disease Stage at Presentation
}

\author{
Afshan Zahedi, ${ }^{1}$ Louis Bondaz, ${ }^{2}$ Murali Rajaraman, ${ }^{3}$ William D. Leslie, ${ }^{4}$ Cheryl Jefford, ${ }^{5,6}$ \\ James Edward Young, ${ }^{7}$ K. Alok Pathak, ${ }^{8}$ Yves Bureau, ${ }^{9}$ Irina Rachinsky, ${ }^{10}$ M. Badreddine, ${ }^{10}$ \\ Sarah De Brabandere, ${ }^{10}$ Helen Fong, ${ }^{3}$ Anastasios Maniakas, ${ }^{11}$ and Stan Van Uum ${ }^{12}$
}

Background: Well-differentiated thyroid cancer (DTC) presents at a more advanced stage in men than in women, and the mortality in men is higher than that in women. However, it is not clear whether DTC recurrence is affected by sex independent of stage at presentation. The objective of the present study was to assess if male sex is an independent risk factor for recurrence of DTC.

Methods: The Canadian Collaborative Network for Cancer of the Thyroid (CANNECT) is a collaborative registry to describe patterns of care for thyroid cancer. We included patients from the CANNECT registry with DTC diagnosed at age 18 or older between 2000 and 2010. We compared men and women with respect to presentation, management, and recurrence risk, stratified for American Joint Committee on Cancer (AJCC) stage.

Results: We included 2595 patients, $2067(79.7 \%)$ women and $528(20.3 \%)$ men. Men presented with more advanced AJCC stage $(p<0.001)$, T stage $(p<0.001)$, N stage $(p<0.001)$, and M stage $(p=0.002)$ There was no difference in follow-up duration between women $(7.7 \pm 4.0$ [mean \pm standard deviation] years) and men $(7.7 \pm 4.0$ years, $p=0.985)$. Overall recurrence was $2.2 \%(n=46)$ for women and $8.5 \%(n=45)$ for men $(p<0.001)$. In multivariate analysis adjusted for AJCC stage, men were at significantly greater risk for DTC recurrence than women (adjusted hazard ratio 2.72 [95\% confidence interval [CI] 1.78-4.20]; $p<0.001$ ). In multivariate analysis adjusted for tumor-node-metastasis (TNM) stage, men were at significantly greater risk for DTC recurrence than women (adjusted hazard ratio 2.31 [CI 1.48-3.60]; $p<0.001$ ).

Conclusions: Our study confirms that the risk for recurrence of DTC is higher in men than in women. Although men tend to present with more advanced-stage disease, the difference in recurrence risk persists when adjusted for stage of presentation. It needs to be determined whether sex should influence follow-up intensity and/or duration.

Keywords: thyroid cancer, sex, recurrence, risk stratification, follow-up

\section{Introduction}

W ELL-DIFFERENTIATED THYROID CANCER (DTC) is the most common thyroid malignancy comprising over $90 \%$ of all thyroid cancers (1). The overall five-year survival of DTC is excellent at $97.9 \%$, and that of low risk (stages I and II) being almost $100 \%$ (2). It is well known that thyroid cancer presents in more advanced stage in men than in women (3), and the mortality in men is higher than that in women (4).
Over the last decade, there is increased focus on the risk for recurrence of DTC (5), as risk stratification impacts treatment decisions regarding target thyrotropin levels and administration of radioactive iodine (RAI). The 2015 American Thyroid Association (ATA) guidelines describe various factors that affect the risk of structural recurrence, some of which are extrathyroidal extension (ETE), lymph node involvement, tumor multifocality, and BRAF mutation status (6). Patient sex is not listed as a factor affecting recurrence risk. The impact of sex on DTC recurrence risk is unclear and

\footnotetext{
${ }^{1}$ Department of Medicine, Women's College Hospital, Toronto, Canada.

${ }^{2}$ Hopital Maisonneuve-Rosemont, ${ }^{11}$ Department of Surgery, Université de Montréal, Montreal, Canada.

${ }^{3}$ Department of Radiation Oncology, Dalhousie University, Halifax, Canada.

Departments of ${ }^{4}$ Internal Medicine and ${ }^{8}$ Surgery, University of Manitoba, Winnipeg, Canada.

Departments of ${ }^{5}$ Radiology and ${ }^{6}$ Nuclear Medicine, Memorial University, St. John's, Canada.

${ }^{7}$ Department of Surgery, McMaster University, Hamilton, Canada.

Departments of ${ }^{9}$ Imaging and ${ }^{12}$ Medicine, Western University, London, Ontario, Canada.

${ }^{10}$ Department of Imaging, Division of Nuclear Medicine, Western University, London, Ontario, Canada.
} 
studies on this topic are limited. The objective of the present study was to assess if male sex is an independent risk factor for recurrence of DTC.

\section{Materials and Methods}

\section{Patient/participating centers}

Patients were recruited from thyroid cancer clinics at six Canadian centers that participate in the Canadian Collaborative Network for Cancer of the Thyroid (CANNECT); a collaborative registry to describe patterns of care for thyroid cancer across Canada. To participate, a center had to have enrolled at least 30 patients. The centers include Fredericton, New Brunswick (NB); Halifax, Nova Scotia (NS); Hamilton, Ontario (ON); London, ON; Toronto, ON; and Winnipeg, Manitoba (MB). All centers are regional referral centers, with the exception for CancerCare Manitoba, which collects information from all thyroid cancer patients within the entire province. Some centers (London, Winnipeg, and Toronto) had data available starting in 2000 , while other centers started to collect data in later years. Patients were included if they were diagnosed between 2000 and 2010, had welldifferentiated (papillary or follicular) thyroid cancer diagnosed at age 18 or older, with information on presentation, treatment, and outcomes; patients with incomplete data and patients with persistent disease after initial surgery were excluded. In total, we excluded 327 patients (11\%) because of incomplete data and/or loss to follow-up. We did not include patients with poorly differentiated histology. We did include aggressive variants such as tall cell and columnar variants and grouped them together as aggressive variants. Patient with metastatic disease at presentation were only included if they had become disease free after initial management.

Each participating center received study approval by the research ethics boards at their respective institutions.

\section{Data collection}

All centers collected core data elements related to thyroid cancer diagnosis and treatment. The core data elements included year of diagnosis, age at diagnosis, sex, initial surgical management, pathology, staging, and RAI administration and outcomes (recurrence, persistent disease, and status at last follow-up).

Cases were staged according to the American Joint Committee on Cancer (AJCC) seventh edition and by tumor-nodemetastasis (TNM) classification system (sixth edition). Information pertaining to clinical and pathological stage of thyroid cancer at presentation was obtained from clinic notes prepared by treating physicians and cross-referenced with original pathology and/or imaging reports. All data were deidentified to protect patients' privacy and confidentiality, and submitted for central data analysis. The data were carefully verified and checked for potential duplicates to avoid including the same patient more than once across multiple centers.

\section{Ascertainment of recurrence}

Recurrence was based on clinical, biochemical, pathological, and structural information. Recurrence was only diagnosed after being considered disease free following initial management. Definitions varied slightly between centers, and details are provided in Supplementary Table S1. Importantly, for each participating center, the definition of recurrence was the same for men and women.

\section{Data analysis}

Descriptive data are presented as mean \pm standard deviation (SD) or percentage as appropriate. Time to recurrence curves were calculated with the Kaplan-Meier method and analyzed using log-rank testing. Univariate analysis was performed using the chi-square test to compare distribution, and one-way analysis of variance to compare means of continuous variables. Association assessment was done using chi-square testing. Multivariate analysis on differences in treatment between men and women was performed using regression analysis. Recurrence hazard was analyzed with multivariate Cox regression using SPSS version 24 (IBM Corporation, Armonk, NY). Statistical significance was accepted at a $p$-value $<0.05$.

\section{Results \\ Patients and participating centers}

In total, we included 2595 patients with DTC, 2067 (79.7\%) women and 528 (20.3\%) men. Baseline characteristics are shown in Table 1. At presentation, men tended to be slightly older than women $(50.8 \pm 14.3$ vs. $46.8 \pm 14.0$ years, $p<0.001$ ), and more often underwent neck dissection (37.9\% vs. $28.5 \%, p<0.001)$. Follicular thyroid cancer was more common in men $(9.3 \%)$ than in women $(5.7 \%, p=0.002)$, and ETE was also more frequent in men $(18.1 \%)$ than in women $(9.3 \%, p<0.001)$. Overall, men presented with more advanced AJCC stage $(p<0.001)$, T stage $(p<0.001)$, N stage $(p<0.001)$, and M stage $(p=0.002)$, and RAI was given more often in men than in women $(72.2 \%$ vs. $64.5 \%$, $p=0.001)$.

\section{Follow-up and risk for recurrence}

There was no difference in follow-up duration between men ( $7.7 \pm 4.0$ years) and women $(7.7 \pm 4.0$ years, $p=0.985)$. Recurrence of DTC occurred in $8.5 \%(n=45)$ of men versus $2.2 \%(n=46)$ of women $(p<0.001)$. After stratifying for AJCC stage, men were more likely to have DTC recurrence for stages I, II, and III, but not for stage IV (Table 2). After stratifying for T stage, recurrence occurred more often in men than in women for stages $\mathrm{T} 1, \mathrm{~T} 2$, and $\mathrm{T} 3$, but not for stage $\mathrm{T} 4$ (Table 2). We also stratified by AJCC stage and by age (younger/older than 55 years). The results are shown in Supplementary Table S2. For the group younger than 55 years, the recurrence rate was lower for women than for men for stages I and II (both $p<0.001$ ), but not for stages III and IV. For the group older than 55 years, the recurrence rate was lower for women than for men for stages I $(p<0.001)$ and III $(p=0.035)$, but not for stages II and IV.

Kaplan-Meier analysis for DTC recurrence, stratified by AJCC stage, shows decreased recurrence-free survival in men compared with women for AJCC stages I, II, and III, but not for stage IV (Fig. 1). Kaplan-Meier curves for DTC recurrence, stratified by $\mathrm{T}$ stage, show that recurrence-free survival is decreased in men compared with women for T1, T2, and T3 stages, but not for T4 stage (Fig. 2). 
Table 1. Baseline Characteristics and Outcomes According to Sex

\begin{tabular}{|c|c|c|c|c|}
\hline Variables & Total $(\mathrm{n}=2595)$ & Women $(\mathrm{n}=2067)$ & $\operatorname{Men}(\mathrm{n}=528)$ & $\mathrm{p}-$ Value $^{\mathrm{a}}$ \\
\hline \multicolumn{5}{|l|}{ Demographic } \\
\hline Age at diagnosis (years, mean \pm SD) & $47.6 \pm 14.1$ & $46.8 \pm 14.0$ & $50.8 \pm 14.3$ & $<0.001$ \\
\hline \multicolumn{5}{|l|}{ Thyroid surgical type } \\
\hline Total thyroidectomy ${ }^{\mathrm{b}}$ & $2256(86.9 \%)$ & $1785(86.4 \%)$ & $471(89.2 \%)$ & \multirow[t]{2}{*}{0.083} \\
\hline Hemithyroidectomy & $339(13.1 \%)$ & $282(13.6 \%)$ & $57(10.8 \%)$ & \\
\hline Neck dissection $^{\mathrm{c}}$ & $790(30.4 \%)$ & $590(28.5 \%)$ & $200(37.9 \%)$ & $<0.001$ \\
\hline \multicolumn{5}{|l|}{ Pathology } \\
\hline Papillary & $2429(93.6 \%)$ & $1950(94.3 \%)$ & $479(90.7 \%)$ & \multirow[t]{2}{*}{0.002} \\
\hline Follicular & $166(6.4 \%)$ & $117(5.7 \%)$ & $49(9.3 \%)$ & \\
\hline ETE & $292(11.2 \%)$ & $196(9.5 \%)$ & $96(18.1 \%)$ & $<0.001$ \\
\hline Lymphovascular invasion & $73(2.8 \%)$ & $57(2.8 \%)$ & $16(3.0 \%)$ & 0.739 \\
\hline \multicolumn{5}{|l|}{ T stage } \\
\hline $\mathrm{T} 1$ & $1286(49.5 \%)$ & $1095(52.9 \%)$ & $191(36.1 \%)$ & \multirow[t]{4}{*}{$<0.001$} \\
\hline $\mathrm{T} 2$ & $701(27.0 \%)$ & $575(27.8 \%)$ & $126(23.9 \%)$ & \\
\hline $\mathrm{T} 3$ & $547(21.1 \%)$ & $361(17.5 \%)$ & $186(35.2 \%)$ & \\
\hline $\mathrm{T} 4$ & $61(2.3 \%)$ & $36(1.7 \%)$ & $25(4.7 \%)$ & \\
\hline \multicolumn{5}{|l|}{$\mathrm{N}$ stage } \\
\hline N0, Nx & $2096(80.8 \%)$ & $1727(83.6 \%)$ & $369(69.9 \%)$ & \multirow[t]{3}{*}{$<0.001$} \\
\hline Nla & $296(11.4 \%)$ & $220(10.6 \%)$ & $76(14.4 \%)$ & \\
\hline $\mathrm{N} 1 \mathrm{~b}$ & $203(7.8 \%)$ & $120(5.8 \%)$ & $83(15.7 \%)$ & \\
\hline \multicolumn{5}{|l|}{ M stage } \\
\hline M0 & $2574(99.2 \%)$ & $2056(99.5 \%)$ & $518(97.9 \%)$ & \multirow[t]{2}{*}{0.002} \\
\hline M1 & $21(0.8 \%)$ & $11(0.5 \%)$ & $10(1.9 \%)$ & \\
\hline \multicolumn{5}{|l|}{ AJCC stage } \\
\hline I & $1796(69.2 \%)$ & $1511(73.1 \%)$ & $285(53.9 \%)$ & \multirow[t]{4}{*}{$<0.001$} \\
\hline II & $327(12.6 \%)$ & $256(12.4 \%)$ & $71(13.4 \%)$ & \\
\hline III & $344(13.2 \%)$ & $232(11.2 \%)$ & $112(21.2 \%)$ & \\
\hline IV & $128(4.9 \%)$ & $68(3.3 \%)$ & $60(11.3 \%)$ & \\
\hline Radioactive iodine & $1715(66.1 \%)$ & $1334(64.5 \%)$ & $381(72.2 \%)$ & 0.001 \\
\hline \multicolumn{5}{|l|}{ Follow-up } \\
\hline Duration (years, mean \pm SD) & $7.7 \pm 4.0$ & $7.7 \pm 4.0$ & $7.7 \pm 4.0$ & 0.985 \\
\hline Recurrence & $91(3.5 \%)$ & $46(2.2 \%)$ & $45(8.5 \%)$ & $<0.001$ \\
\hline
\end{tabular}

We performed multivariate analysis for DTC recurrence with AJCC staging using Cox proportional hazards regression (Table 3). Male sex was an independent predictor for recurrence, with AJCC stage-adjusted hazard ratio $2.72(95 \%$ confidence interval [CI 1.78-4.20]; $p<0.001)$. Other significant predictors for DTC recurrence were AJCC stage IV and the presence of ETE and of follicular pathology. After adjustment for sex, age older than 55 years was not a significant predictor of recurrence (hazard ratio 1.21 [CI 0.74-1.99], $p=0.452$ ). We analyzed if sex modified the TNM stageassociated effect on recurrence. Cox regression with TNM staging found that male sex was again an independent risk factor for recurrence with an adjusted hazard ratio of 2.31 $(1.48-3.46 ; p<0001)$. Age older than 55 years at diagnosis, stages T3 and T4, N stage, and follicular pathology were significant independent predictors of recurrence (Table 4). We also assessed recurrence risk after adjusting for age and $\mathrm{T}$ stage (Table 5). For the group younger than 55 years, recurrence risk for men was significantly higher for $\mathrm{T} 2$ and $\mathrm{T} 3$, and for the group older than 55 years, the recurrence risk for men was significantly higher than for women for stages T1 and T3.
Table 2. Differentiated Thyroid Cancer Recurrence Risk, Women Versus Men, SPECIFIED FOR AmERICAN JoINT COMmitTeE on Cancer Stage and T Stage

\begin{tabular}{|c|c|c|c|}
\hline & Women (\%) & Men (\%) & $\mathrm{p}-$ Value $^{\mathrm{a}}$ \\
\hline Total & $46(2.2)$ & $45(8.5)$ & $<0.001$ \\
\hline \multicolumn{4}{|c|}{ AJCC stage } \\
\hline I & $25 / 1511(1.7)$ & $19 / 285(6.7)$ & $<0.001$ \\
\hline II & $2 / 256(0.8)$ & $5 / 71(7.0)$ & 0.001 \\
\hline III & $7 / 232(3.0)$ & $11 / 112(9.8)$ & 0.008 \\
\hline IV & $12 / 68(17.6)$ & $10 / 60(16.7)$ & 0.883 \\
\hline \multicolumn{4}{|c|}{$\mathrm{T}$ stage } \\
\hline $\mathrm{T} 1^{\circ}$ & 9/1095 (0.8) & $6 / 191(3.2)$ & 0.006 \\
\hline $\mathrm{T} 2$ & $9 / 575$ (1.6) & $8 / 126(6.3)$ & 0.002 \\
\hline T3 & $18 / 361(5.0)$ & $24 / 186(12.9)$ & $<0.001$ \\
\hline $\mathrm{T} 4$ & $10 / 36(27.8)$ & $7 / 25(28.0)$ & 0.985 \\
\hline
\end{tabular}

${ }^{\mathrm{a} C h i}$-square test. 

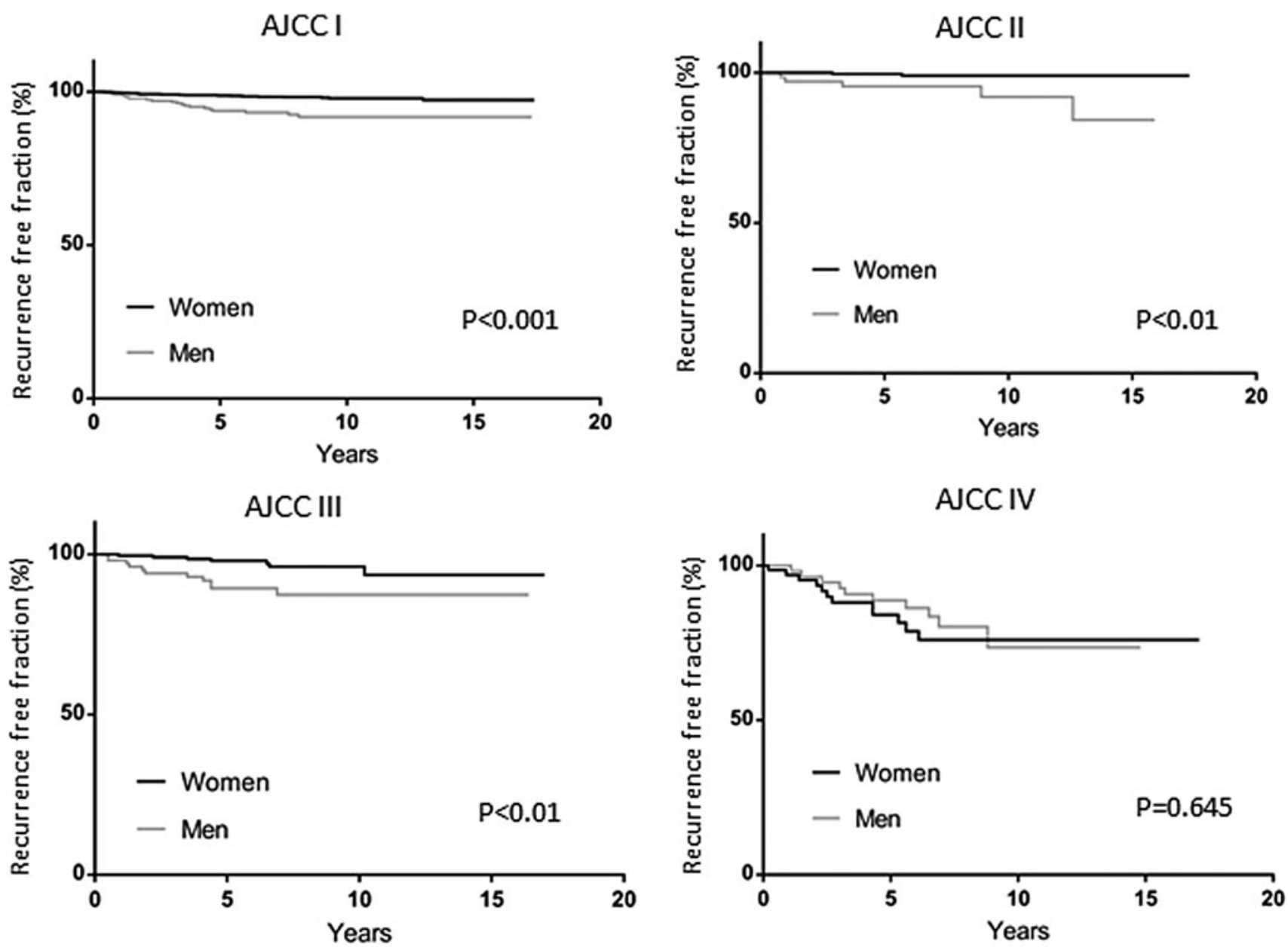

FIG. 1. Risk for recurrence of differentiated thyroid cancer comparing men and women, by AJCC stage. Comparison of survival curves was completed using log-rank (Mantel-Cox) test. AJCC, American Joint Committee on Cancer.

\section{Discussion}

In this study of patients with DTC, we found that recurrence risk was higher in men than in women, even when adjusted for stage at presentation and other factors that were found to be predictors of DTC recurrence risk. The recurrence risk was more than 3-fold higher for men presenting with AJCC stages I, II, and III and T stages 1 and 2, and about 2.5-fold for patients with T3 stage. In contrast, there was no statistically significant difference in recurrence risk between men and women for AJCC stage IV and T4 stage.

The present study is consistent with other studies showing that men tend to present with more advanced disease $(3,4,7,8)$. Disease-specific survival is worse in men, especially in the younger age groups (4), but this might not be true when disease severity is considered (7).

Our findings differ from the study by Toniato et al. who did not find an effect of sex on recurrence risk in 950 patients with papillary thyroid cancer diagnosed between 1990 and 2005 (9). In 1979, Byar et al. (10) reported increased recurrence risk in men compared with women. Both the study by Toniato et al. and by Byar et al. did not differentiate between persistent and recurrent disease, which may have masked a sex effect on recurrence as was found in our study. Thus, we suggest that the studies by Toniato et al. and Byar et al. represent a mixed rate of persistent/recurrent disease, while our study provides an estimate of true recurrence. A recent study by Sapuppo et al. (11) showed that the female to male ratio is lower in persistent disease (1.9/1) than in recurrent disease (4.8/1), and the authors emphasize that it is important to differentiate between persistent and recurrent disease. At this time, there is no explanation to the differences in disease presentation according to sex (12).

In the present study, men underwent lymph node dissection more frequently than women, and also received RAI more frequently. More aggressive initial treatment in men than in women was also reported in the study by Nilubol et al. (7). If anything, this more aggressive treatment would be expected to result in lower, not higher, recurrence rates in men.

The 2015 ATA guidelines call for improved risk stratification to improve the prognostic predictive ability of the AJCC/UICC TNM staging system (6). The guidelines list several potential variables, including the specific histology, molecular profile, size and location of distant metastases, functional status of the metastases, and effectiveness of initial therapy. However, the guidelines do not mention sex as a predictor and neither did the 2009 ATA guidelines (5). Based on our study, we suggest that sex should be considered a potential additional variable for improving overall risk stratification. The AJCC staging systems were not 
T1

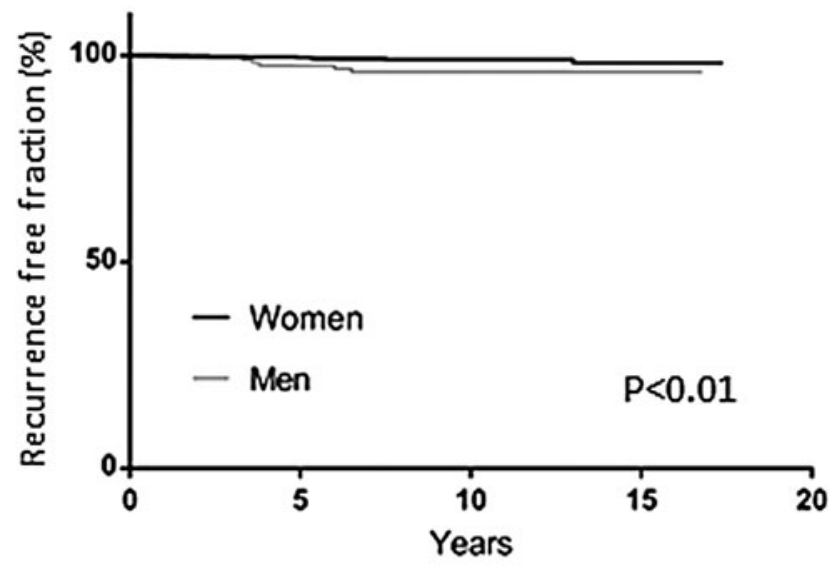

T3

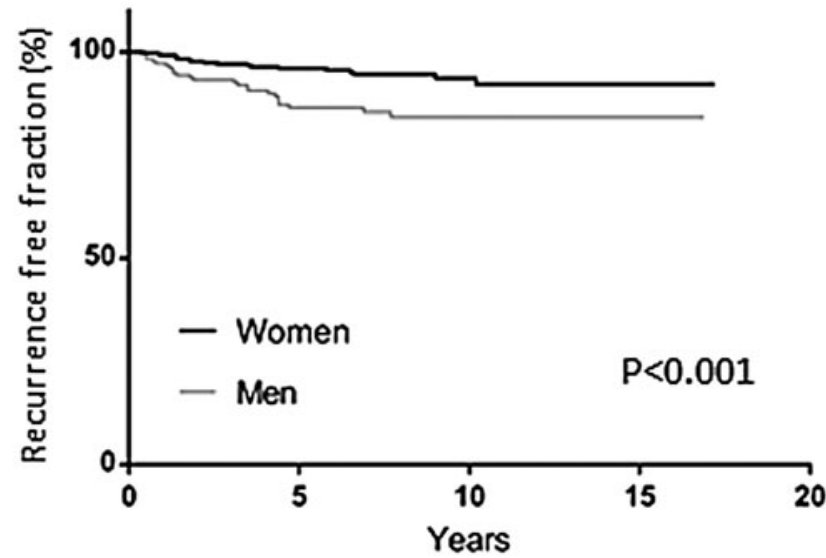

$\mathrm{T} 2$

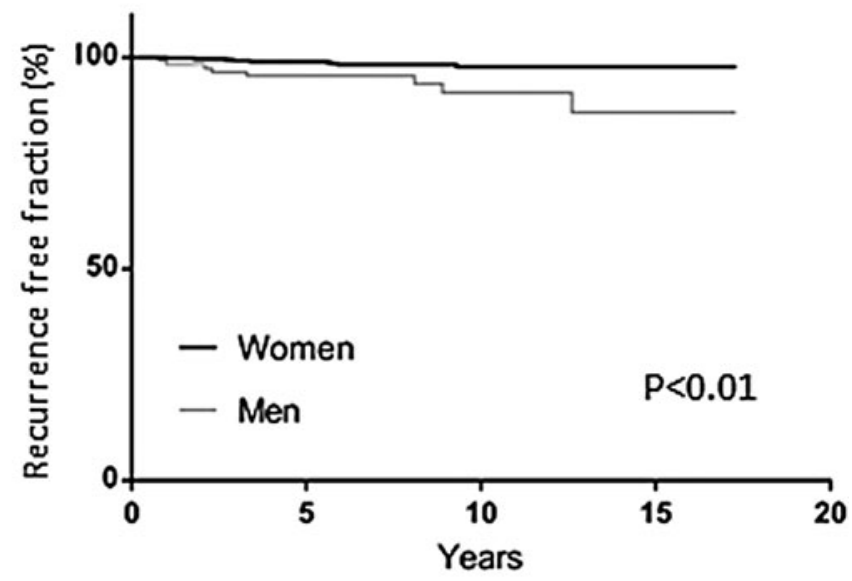

T4

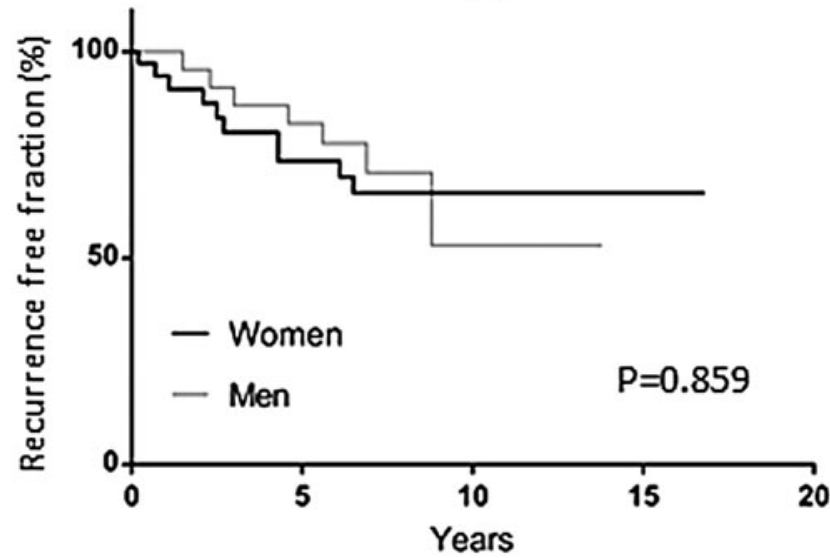

FIG. 2. Risk for recurrence of differentiated thyroid cancer comparing men and women, by tumor (T) stage. Comparison of survival curves was completed using log-rank (Mantel-Cox) test.

developed as a predictor of recurrence risk. Despite this, it is interesting that after adjustment for sex, age no longer is a predictor of recurrence risk for AJCC-based recurrence risk, but remains an independent predictor for $\mathrm{T}$ stage-based recurrence risk.

There are several limitations to our study. These include its historical nature, and the lack of information on some tumor

\begin{tabular}{lcr}
\multicolumn{2}{c}{ Table 3. Cox Regression for Recurrence Hazard } \\
With American Joint Committee on Cancer Staging \\
\hline Risk factor & Hazard ratio $[$ CI] & p-Value \\
\hline Sex (men) & $2.72[1.78-4.20]$ & $<0.001$ \\
Age >55 years & $1.21[0.74-1.99]$ & 0.452 \\
AJCC & Referent & \\
I & $0.78[0.34-1.78]$ & 0.556 \\
II & $1.45[0.78-2.71]$ & 0.239 \\
III & $3.47[1.76-6.85]$ & $<0.001$ \\
IV & $2.48[1.47-4.19]$ & 0.001 \\
ETE present & $2.00[1.14-3.50]$ & 0.016 \\
Follicular pathology &
\end{tabular}

AJCC stage I was referent for stages II, III, and IV; papillary thyroid cancer was referent for follicular thyroid cancer. All twoway interactions with sex were nonsignificant ( $p$-interaction $\geq 0.1$ ). CI, $95 \%$ confidence interval. features, including presence of multiple DTC tumors, BRAF mutations, and vascular invasion; these were data that were not commonly assessed between 2000 and 2010. Recurrence was based on a combination of structural and biochemical data, and definitions varied slightly between the participating

Table 4. Cox Regression for Recurrence Hazard With Tumor-Node-Metastasis Staging

\begin{tabular}{lcr}
\hline Risk factor & Hazard ratio $[$ CI] & p-Value \\
\hline Sex (men) & $2.31[1.48-3.60]$ & $<0.001$ \\
Age $\geq 55$ year & $1.63[1.04-2.56]$ & 0.033 \\
T & Referent & \\
T1 & $1.74[0.86-3.52]$ & 0.121 \\
T2 & $4.26[2.23-8.17]$ & $<0.001$ \\
T3 & $8.17[3.38-19.71]$ & $<0.001$ \\
T4 & Referent & \\
Nx/0 & $2.19[1.18-4.05]$ & 0.013 \\
N1a & $3.66[2.09-6.40]$ & $<0.001$ \\
N1b & $0.95[0.53-1.71]$ & 0.867 \\
ETE present & $2.1[1.17-3.84]$ & 0.013 \\
Follicular pathology & \\
\hline
\end{tabular}

Stage $\mathrm{T} 1$ was referent for stages $\mathrm{T} 2, \mathrm{~T} 3$, and $\mathrm{T} 4 ; \mathrm{Nx} / 0$ was referent for N1a and N1b; papillary thyroid cancer was referent for follicular thyroid cancer. All two-way interactions with sex were nonsignificant ( $p$-interaction $\geq 0.1$ ). 
Table 5. Differentiated Thyroid Cancer Recurrence Risk, Women Versus Men, Specified for T Stage and Age Older/Younger Than 55 Years

\begin{tabular}{|c|c|c|c|c|c|c|}
\hline \multirow[b]{2}{*}{ Age } & \multicolumn{3}{|c|}{$<55$ years } & \multicolumn{3}{|c|}{$\geq 55$ years } \\
\hline & Women (\%) & Men (\%) & $\mathrm{p}-$ Value $e^{\mathrm{a}}$ & Women (\%) & Men (\%) & $\mathrm{p}-$ Value $e^{\mathrm{a}}$ \\
\hline Total & $30 / 1456(2.1)$ & $23 / 281(8.2)$ & $<0.001$ & $16 / 566(2.8)$ & $22 / 203(10.8)$ & $<0.001$ \\
\hline \multicolumn{7}{|l|}{$\mathrm{T}$ stage } \\
\hline 1 & $7 / 770(0.9)$ & 2/108 (1.9) & 0.369 & 2/316 (0.6) & 4/77 (5.2) & 0.004 \\
\hline 2 & $6 / 445(1.3)$ & $6 / 77(7.8)$ & $<0.001$ & $3 / 122(2.5)$ & $2 / 42(4.8)$ & 0.470 \\
\hline 3 & $13 / 224(5.8)$ & $13 / 86(15.1)$ & $<0.001$ & $5 / 119(4.2)$ & $11 / 76(14.5)$ & 0.020 \\
\hline 4 & $4 / 17(23.5)$ & $2 / 10(20.7)$ & 0.865 & 6/9 (66.7) & $5 / 8(62.5)$ & 0.934 \\
\hline
\end{tabular}

${ }^{\mathrm{a}}$ Chi-square test.

centers. However, within each center, the definition of recurrence was the same for men and women. Furthermore, we used AJCC 7 for our classification, which is slightly different from the AJCC 8 classification that has recently been published (13), which may slightly impact the generalizability of our results to future patients. Since patients were diagnosed between 2000 and 2010, the treatment choices reflect the treatment at that time, and may be slightly different from those recommended in the 2015 ATA guidelines. This may impact the ability to generalize our findings to patients currently being diagnosed and treated. In addition, the exclusion of $11 \%$ of patients due lack of data and/or loss of follow-up may have created a further selection bias. We do not know how many of the follicular thyroid cancer tumors are minimally invasive, and our data do not allow us to reassess that. Therefore, we are not able to determine how many of our patients would potentially have NIFTP. At the time of diagnosis, these were considered FTC and were treated accordingly. Also, our study did not include information on the socioeconomic status of participating patients. In our models, we did not correct for neck dissection at the time of initial management. However, neck dissection was performed more frequently in men than in women so that our estimates for recurrence in relation to sex are likely conservative. Our study has several strengths, including its large number of patients from multiple centers across Canada, and a median follow-up duration of more than seven years.

Overall, the results of the present study suggest that sex should be taken into consideration when evaluating risk of recurrence for DTC. If confirmed by other studies, sex may have implications for initial management and intensity and/or duration of follow-up.

\section{Acknowledgments}

We thank Angela McGibbon, $\mathrm{MD}, \mathrm{PhD}$, deceased, for her contributions to this study.

We thank all the patients who participated in this study.

We thank the Canadian Collaborative Network for Cancer of the Thyroid (CANNECT) group members:

Ralf Paschke and Dean Reuther (Calgary, Alberta). Formerly Angela McGibbon (Fredericton, New Brunswick). Murali (Mal) Rajaraman and Helen Fong; Inactive Members: Ali Imran, Martin Bullock, Steve Burrell, and Tim Wallace (Halifax, Nova Scotia). J.E. (Ted) Young, Jesse Pasternak, and John Paul Oliveria (Hamilton, Ontario). Josh Lakoff and Kim Suffron (Kingston, Ontario). Stan Van
Uum, Sarah DeBrabandere, Mahmoud Baddredine, and Irina Rachinsky; Inactive Members: Deric Morrison, Ranjit Singarayer, and Terri Paul (London, Ontario). Louis Bondaz and Ainsley Dawson (Montreal, Quebec [Hôpital MaisonneuveRosemont]). Andree Boucher, Anastasios (Tasso) Maniakas, Hortensia Mircescu, and Rebecca LeBeouf; Inactive Members: Bernard L'Esperance (Montreal, Quebec [CHUM, Hôpital Notre-Dame]). Cheryl Jefford and Michelle Simms; Inactive Members: Cathy Murray and Carol Joyce (St. John's, Newfoundland). Afshan Zahedi; Inactive Members: Catherine Kelly and Rosaria Briones-Urbina (Toronto, Ontario [Women's College and Finch]). Jesse Pasternak (Toronto, Ontario [UHN]). William D. Leslie, K. Alok Pathak (Winnipeg, Manitoba [registry based]).

\section{Author Disclosure Statement}

M.R., I.R., and S.V.U. have served on the scientific advisory boards for Sanofi-Genzyme. M.R., I.R., J.E.Y., and S.V.U. have served on the scientific advisory boards for Eisai Limited. A.Z., L.B., W.D.L., C.J., A.M., K.A.P., Y.B., M.B., S.D.B., H.F., and A.M. have nothing to disclose.

\section{Funding Information}

CANNECT has received funding in the form of unrestricted grants from Genzyme Inc., Canada.

\section{Supplementary Material}

Supplementary Table S1

Supplementary Table S2

\section{References}

1. Jemal A, Siegel R, Ward E, Murray T, Xu J, Smigal C, Thun MJ 2006 Cancer statistics, 2006. CA Cancer J Clin 56:106-130.

2. Tsang RW, Brierley JD, Simpson WJ, Panzarella T, Gospodarowicz MK, Sutcliffe SB 1998 The effects of surgery, radioiodine, and external radiation therapy on the clinical outcome of patients with differentiated thyroid carcinoma. Cancer 82:375-388.

3. Machens A, Hauptmann S, Dralle H 2006 Disparities between male and female patients with thyroid cancers: sex difference or gender divide? Clin Endocrin 65:500-505.

4. Jonklaas J, Nogueras-Gonzalez G, Munsell M, Litofsky D, Ain KB, Bigos ST, Brierley JD, Cooper DS, Haugen BR, Ladenson PW, Magner J, Robbins J, Ross DS, Skarulis 
MC, Steward DL, Maxon HR, Sherman SI 2012 The impact of age and gender on papillary thyroid cancer survival. J Clin Endocrinol Metab 97:E878-E887.

5. Cooper DS, Doherty GM, Haugen BR, Kloos RT, Lee SL, Mandel SJ, Mazzaferri EL, McIver B, Pacini F, Schlumberger M, Sherman SI, Steward DL, Tuttle RM 2009 Revised American Thyroid Association management guidelines for patients with thyroid nodules and differentiated thyroid cancer. Thyroid 19:1167-1214.

6. Haugen BR, Alexander EK, Bible KC, Doherty GM, Mandel SJ, Nikiforov YE, Pacini F, Randolph GW, Sawka AM, Schlumberger M, Schuff KG, Sherman SI, Sosa JA, Steward DL, Tuttle RM, Wartofsky L 20162015 American Thyroid Association management guidelines for adult patients with thyroid nodules and differentiated thyroid cancer: The American Thyroid Association guidelines task force on thyroid nodules and differentiated thyroid cancer. Thyroid 26:1-133.

7. Nilubol N, Zhang L, Kebebew E 2013 Multivariate analysis of the relationship between male sex, disease-specific survival, and features of tumor aggressiveness in thyroid cancer of follicular cell origin. Thyroid 23:695-702.

8. Oyer SL, Smith VA, Lentsch EJ 2013 Sex is not an independent risk factor for survival in differentiated thyroid cancer. Laryngoscope 123:2913-2919.

9. Toniato A, Boschin I, Casara D, Mazzarotto R, Rubello D, Pelizzo M 2008 Papillary thyroid carcinoma: factors influencing recurrence and survival. Ann Surg Oncol 15: 1518-1522.

10. Byar DP, Green SB, Dor P, Williams ED, Colon J, van Gilse HA, Mayer M, Sylvester RJ, van Glabbeke M 1979 A prognostic index for thyroid carcinoma. A study of the E.O.R.T.C. Thyroid Cancer Cooperative Group. Eur J Cancer 15:1033-1041.

11. Sapuppo G, Tavarelli M, Belfiore A, Vigneri R, Pellegriti G 2019 Time to separate persistent from recurrent differentiated thyroid cancer: different conditions with different outcomes. J Clin Endocrinol Metab 104:258-265.

12. Rahbari R, Zhang L, Kebebew E 2010 Thyroid cancer gender disparity. Future Oncol 6:1771-1779.

13. Amin MB ES, Greene FL, et al. (eds) 2017 AJCC Cancer Staging Manual. Eighth edition. Springer, New York.

Address correspondence to:

Stan Van Uum, MD, PhD

St. Joseph's Health Care

Room B4-130,

268 Grosvenor Street

London N6A $4 \mathrm{~V} 2$

Ontario

Canada

E-mail: stan.vanuum@sjhc.london.on.ca 